PROCEEDINGS OF THE

AMERICAN MATHEMATICAL SOCIETY

Volume 130, Number 7, Pages 1923-1925

S 0002-9939(01)06400-0

Article electronically published on November 15, 2001

\title{
ABELIAN POINT STABILIZERS IN TRANSITIVE PERMUTATION GROUPS
}

\author{
I. M. ISAACS
}

(Communicated by Stephen D. Smith)

\begin{abstract}
In this note we prove that if the point stabilizer $A$ in a transitive permutation group of degree $m$ is abelian, then the exponent of $A$ is less than $m$. This extends an earlier result of Andrea Lucchini, who proved this in the case where $A$ is cyclic.
\end{abstract}

The following pretty result of Andrea Lucchini appears in [2.

Theorem (Lucchini). Suppose that $A$ is a point stabilizer in a transitive permutation group of degree $m>1$. If $A$ is cyclic, then $|A|<m$.

In this brief note, we use some of Lucchini's methods to prove a somewhat stronger result.

Theorem A. Suppose that $A$ is a point stabilizer in a transitive permutation group of degree $m>1$. If $A$ is abelian, then the exponent $\exp (A)<m$.

Lucchini's proof depends on a theorem of A. Delgado and A. Chermak [1], but we prefer to use the somewhat similar (but more powerful) result of V. I. Zenkov [3], which we state here for the convenience of the reader. (We mention that the proof of Zenkov's theorem is quite short, and it does not rely on the classification of simple groups.)

Theorem (Zenkov). Let $A$ and $B$ be abelian subgroups of a finite group $G$. Then there exists an element $g \in G$ such that $A \cap B^{g} \subseteq \mathbf{F}(G)$, the Fitting subgroup.

We shall also need the following well known fact, which can be viewed as a corollary of Zenkov's theorem. (More is true, however, and even more elementary proofs are available.)

Corollary B. Let $A$ be an abelian group that acts faithfully and coprimely on a nontrivial group $N$. Then $|A|<|N|$.

Proof. Let $G=N A$ be the semidirect product and let $\pi$ be the set of prime divisors of $|A|$. Since $N$ is a $\pi^{\prime}$-group, it follows that $\mathbf{O}_{\pi}(G)$ is contained in the (trivial) kernel of the action of $A$ on $N$. Thus $\mathbf{O}_{\pi}(G)=1$, and we conclude that $A \cap \mathbf{F}(G)=1$. By Zenkov's theorem, therefore, there exists $g \in G$ such that $A \cap A^{g}=1$. Since $A<G$, the subset $A A^{g}$ is not the whole group $G$, and it follows that $|G|>\left|A A^{g}\right|=|A|^{2}$. Thus $|N|=|G: A|>|A|$, as required.

Received by the editors January 30, 2001.

2000 Mathematics Subject Classification. Primary 20B05, 20 D99.

Research partially supported by a grant from the U. S. National Security Agency.

(c)2001 American Mathematical Society 
Proof of Theorem A. Let $G$ be a transitive permutation group of degree $m$ and suppose that a point stabilizer $A$ is abelian. We show by induction on $m$ that $\exp (A) \leq m$ and that this inequality is strict if $m>1$. This assertion is clear when $m=1$, and so we can assume $m>1$. We can also assume, of course, that $A>1$.

If $\mathbf{F}(G)=1$, then by Zenkov's theorem $A \cap A^{g}=1$ for some element $g \in G$. As in the proof Corollary $\mathrm{B}$, it follows that $|G|>\left|A A^{g}\right|=|A|^{2}$, and therefore $\exp (A) \leq|A|<|G: A|=m$, and we are done in this case. We can thus assume that $\mathbf{F}(G)$ is nontrivial, and we choose a minimal normal subgroup $N$ of $G$ such that $N$ is an elementary abelian $p$-group for some prime $p$.

Now $N \nsubseteq A$ since $\operatorname{core}_{G}(A)=1$, and thus $N A>A$. Writing $u=|G: N A|$, we see that $u<m$. Let $K=\operatorname{core}_{G}(N A)$, so that $G / K$ is a transitive permutation group of degree $u$ with point stabilizer $N A / K$. Since $N \subseteq K$, we see that $N A=K A$ and $N A / K=K A / K \cong A /(K \cap A)=A / J$, where we have written $J=K \cap A$. Since $A / J$ is abelian, it follows by the inductive hypothesis that $\exp (A / J) \leq u$, with equality only if $u=1$. Also, we note that $K=N(K \cap A)=N J$ by Dedekind's lemma, and $m / u=|N A: A|=|N: N \cap A|$ is a power of $p$.

First, suppose that $K$ is nonabelian. Write $Z=\mathbf{Z}(K) \triangleleft G$, and note that $Z J$ is abelian since $J$ is abelian and $J \subseteq K$. It follows that $N \nsubseteq Z$, since $N J=K$ is nonabelian. Since $N$ is minimal normal in $G$, we conclude that $N \cap Z=1$.

Now write $V=N Z \cap J$. We have $N \subseteq N Z \subseteq K=N J$, and thus by Dedekind's lemma, $N Z=N(N Z \cap J)=N V$. Also, $N Z$ is abelian, and thus $V$ centralizes both $N$ and $J$. Therefore, $V \subseteq \mathbf{Z}(N J)=\mathbf{Z}(K)=Z \subseteq N Z=N V$. By Dedekind's lemma again, we have $Z=(N \cap Z) V=V \subseteq A$. But $Z \triangleleft G$ and $A$ has trivial core, and it follows that $1=Z=\mathbf{Z}(K)=\mathbf{Z}(N J)$. Since $J$ is abelian, we have $\mathbf{C}_{J}(N)=1$, and $J$ acts faithfully on $N$. In particular, we see that $N \cap A \subseteq \mathbf{C}_{J}(N)=1$, and thus $m / u=|N|$.

Now recall that $N$ is a $p$-group and let $P \in \operatorname{Syl}_{p}(J)$. Then $N P$ is a Sylow $p$ subgroup of $N J=K$, and we note that $K / N$ is abelian since $N A / N$ is abelian. Thus $N P / N \triangleleft G / N$, and so $N P \triangleleft G$, and hence $\mathbf{Z}(N P) \triangleleft G$. But $N \cap \mathbf{Z}(N P)>1$ since $N P$ is a $p$-group, and it follows by the minimality of $N$ that $N \subseteq \mathbf{Z}(N P)$. Thus $P \subseteq \mathbf{C}_{J}(N)=1$, and therefore $J$ is a $p^{\prime}$-group. But $J$ is abelian and acts faithfully on the $p$-group $N$, and so by Corollary B, we have $|J|<|N|=m / u$. Since $\exp (A / J) \leq u$, we have $\exp (A) \leq \exp (A / J)|J|<u(m / u)=m$, and we are done in this case.

We can now assume that $K$ is abelian. We observe that $u>1$, since otherwise $N A=G$ and thus $G=K$ is abelian, which implies that $A=1$, contrary to assumption. We will show that $\exp (J) \leq p$, and thus (since $u>1$ ) we have $\exp (A) \leq \exp (A / J) \exp (J)<u p$. Also, since $1<m / u$ is a power of $p$, we have $p \leq m / u$, and the result will follow.

Let $x \in K=N J$ and write $x=a b$, where $a \in N$ and $b \in J$. Since $K$ is abelian and $N$ is an elementary abelian $p$-group, we have $x^{p}=b^{p} \in J$. Thus $J$ contains the subgroup $\left\{x^{p} \mid x \in K\right\}$, which is characteristic in $K$. Since core $G(A)=1$, this subgroup is trivial, and thus $K$ is an elementary abelian $p$-group. In particular, since $J \subseteq K$, we see that $\exp (J) \leq p$, as claimed, and this completes the proof.

\section{REFERENCES}

1. A. Chermak and A. Delgado, A measuring argument for finite groups, Proc. Amer. Math. Soc. 107 (1989) 907-914. MR 90c:20001 
2. A. Lucchini, On the order of transitive permutation groups with cyclic point-stabilizer, Atti Accad. Naz. Lincei Cl. Sci. Fis. Mat. Natur. Rend. Lincei (9) Mat. Appl. 9 (1998) 241-243. MR 2000k:20004

3. V. I. Zenkov, Intersections of abelian subgroups in finite groups, Math. Notes 56 (1994) 869-871. MR 95m:20021

Department of Mathematics, University of Wisconsin, 480 Lincoln Dr., Madison, WISCONSIN 53706

E-mail address: isaacs@math.wisc.edu 